\title{
Pendra goes Dutch: lessons for the CE mark in Europe
}

Received: 10 February 2005 / Accepted: 24 February 2005 / Published online: 4 May 2005

(C) Springer-Verlag 2005

\begin{abstract}
The development of a truly non-invasive continuous glucose sensor is an elusive goal. We describe the rise and fall of the Pendra device. In 2000 the company Pendragon Medical introduced a truly non-invasive continuous glucose-monitoring device. This system was supposed to work through so-called impedance spectroscopy. Pendra was Conformité Européenne (CE) approved in May 2003. For a short time the Pendra was available on the Dutch direct-to-consumer market. A post-marketing reliability study was performed in six type 1 diabetes patients. Mean absolute difference between Pendra glucose values and values obtained through self-monitoring of blood glucose was 52\%; the Pearson's correlation coefficient was $35.1 \%$; and a Clarke error grid showed $4.3 \%$ of the Pendra readings in the potentially dangerous zone $\mathrm{E}$. We argue that the $\mathrm{CE}$ certification process for continuous glucose sensors should be made more transparent, and that a consensus on specific requirements for continuous glucose sensors is needed to prevent patient exposure to potentially dangerous situations.
\end{abstract}

Keywords Clinical science and care - Devices · Socioeconomic aspects

Abbreviations CE: Conformité Européenne . MAD: mean absolute difference

\footnotetext{
I. M. E. Wentholt · J. B. L. Hoekstra · J. H. DeVries $(\bowtie)$

Department of Internal Medicine,

Academic Medical Center, Meibergdreef 9,

1105 AZ Amsterdam, The Netherlands

e-mail: j.h.devries@amc.uva.nl

Tel.: +31-20-5666525

Fax: $+31-20-5669158$

A. Zwart

U-Diagnostics,

Utrecht, The Netherlands
}

\section{Development and first presentation of a non-invasive continuous glucose sensor}

In 2000 the Swiss company Pendragon Medical presented a new glucose monitoring device called the Pendra. The uniqueness of the Pendra sensor lies in its truly noninvasive character and in its long duration of operation, in contrast to so-called semi-invasive sensors that require subcutaneous insertion and that function for up to $72 \mathrm{~h}$. The sensor is worn on the wrist and resembles a digital watch (Fig. 1). It produces glucose values through a process called impedance spectroscopy. This indirect method of measuring is based on sodium transport over the erythrocyte membrane, which is linked to changes in glucose. These transmembraneous sodium fluxes induce changes in impedance fields. Impedance changes are detected by the Pendra and converted into a glucose value. For many, the essentials of this process are hard to grasp, and it seems unlikely that changes in glucose are the only factor determining changes in impedance. As studies have already demonstrated the influence of erythrocyte sedimentation rate, temperature and haematocrit on impedance parameters, interference by other processes in the body seems a likely possibility [1-3].

The Pendra is equipped with an alarm for upcoming hypoglycaemic or hyperglycaemic conditions.

Data stored in the device can be downloaded onto a computer for more detailed analysis. A drawback of the sensor is the patient-tailored calibration procedure that is required prior to use, lasting 2-3 days. After completing the calibration phase, approximately $30 \%$ of the patients have to stop wearing the sensor, as their skin types and basic skin impedances are apparently unsuitable for the Pendra system [4].

\section{Preparation for the market and CE approval}

Between 2000 and 2004, approximately 20 abstracts on the Pendra system were presented at meetings in the field of 


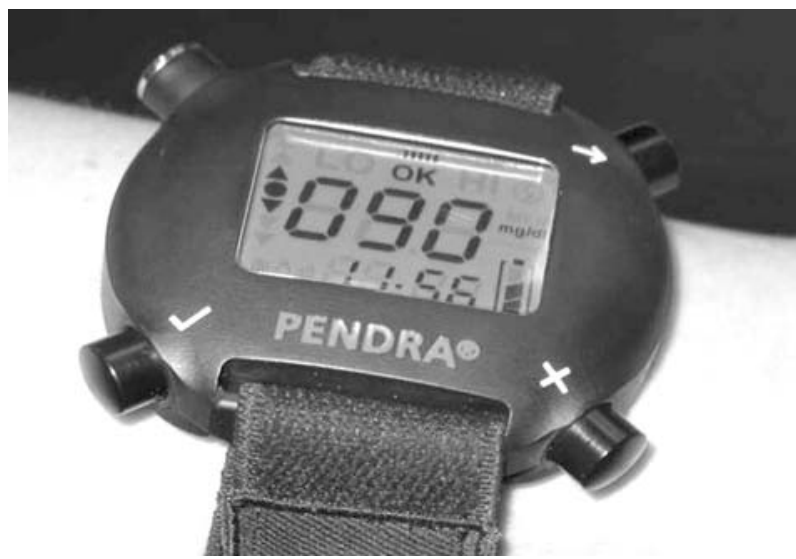

Fig. 1 The Pendra glucose sensor

diabetes. So far, three manuscripts have appeared in peerreviewed journals, none of them dealing with accuracy in diabetes patients [4-6].

On May 12th 2003, Pendra was Conformité Européenne (CE) approved according to the $\mathrm{CE}$ guideline for medical devices CE 93/42, as an adjunctive Type IIb medical device to supplement information obtained through standard glucose monitoring. CE approval allows a company to guarantee and declare that its product meets the relevant requirements as described in the $\mathrm{CE}$ guidelines. The $\mathrm{CE}$ evaluation process consists of two main parts. Firstly, the member states evaluate whether the product is in accordance with the CE essential requirements. For example, one chapter in the essential requirements describes how measuring devices must offer a certain degree of reliability and stability, taking into account their assigned task. Another chapter prescribes that medical devices that are connected to an energy source and report patients' clinical parameters must properly alert for situations that might lead to severe deterioration of the patients' health, e.g. a hypoglycaemic episode in diabetes patients [7]. In the second part of the CE evaluation process, a specialised authority is appointed by the member states in order to make a so-called "valuation of conformity". This authority has to be experienced in evaluating the given type of technology. The valuation includes assessment of conformity to the standards of the quality system applied to the particular product. After approval, it is the responsibility of the authority to ensure that manufacturers follow the standards of the quality system in daily practice [8].

Medical devices are divided into four categories. Pendra's Type IIb category is considered to have great potential risk and therefore requires valuation of conformity in both design and fabric.

After Pendra's CE approval, the company prospered during a financially fruitful period: from May 2003 to June 2004 it received funds from several investors. These funds enabled Pendragon Medical to prepare the launch of the Pendra glucose sensor in a number of European markets in the third quarter of 2004.
Swiss investment company ProgressNow! invest has a controlling interest in Pendragon Medical. After the Pendra $\mathrm{CE}$ approval, the investment company increased its net asset value by $€ 31.60$ to $€ 93.70$ per share [9].

Introduction to the Dutch market and post-calibration reliability of the Pendra device

The Pendra was first inroduced to the market in the Netherlands. Prior to the official launch, and following an internet-based application procedure, selected patients were invited to buy the instrument. The costs were a fee of $€$ 1,250 at the start, followed by a monthly lease price of $€ 95$ for at least two years. These costs were not reimbursed. A patient undergoing treatment at our outpatient clinic informed us of the apparent poor performance of the device he acquired. This experience and the absence of validation studies in diabetes patients prompted us to send out a survey to several clinics in the Netherlands requesting Pendra data and concomitant blood glucose measurements. In total, data from six type 1 diabetes patients (three males) were collected. Mean age was 42.3 years. Each patient had successfully completed the calibration phase. During periods varying from 36 to $96 \mathrm{~h}$, self-control values and corresponding sensor values were noted. All paired values were pooled for further analysis. Pearson's correlation coefficient and mean absolute difference (MAD) compared with capillary blood glucose values were calculated and a Clarke error grid was constructed.

The MAD is the average of all absolute differences, calculated per paired value, between capillary blood glucose value (reference) and sensor glucose value, divided by the corresponding capillary blood glucose value:

$M A D=\sum \frac{\mid \text { Sensor glucose }- \text { Gold standard } \mid}{\text { Gold standard }} * \frac{1}{n} \%$

The Clarke error grid is an established means to evaluate sensor accuracy [10]. It is divided into five different zones. Zones $\mathrm{A}$ and $\mathrm{B}$ represent values that are clinically acceptable for patients with diabetes mellitus. Zone A represents values that differ by no more than $20 \%$ from the reference value and zone B represents values that differ by more than $20 \%$ from the reference value. Results in zones $\mathrm{A}$ and $\mathrm{B}$ will lead to the same treatment decision. Zone C represents values that would result in overcorrecting acceptable glucose values; zone D represents values that are erroneously uncorrected; and zone E represents values that would result in the inverse treatment, e.g. glucose-lowering therapy in reaction to a hypoglycaemic value.

We calculated the number of paired values in each zone of the Clarke error grid using SPSS version 11.5. By using a purpose-built syntax adapted from Clarke et al., problems such as those previously reported concerning the definition of the "upper A-line" were avoided [11].

Correlation between glucose values obtained by the Pendra device and by self-monitoring of blood glucose was 
$35.1 \%$. The MAD (139 paired values) was 52\%. Clarke error grid analysis indicated that $32.4 \%$ of the paired values were in zone A, $46.0 \%$ were in zone $\mathrm{B}, 6.5 \%$ were in zone $\mathrm{C}, 10.8 \%$ were in zone $\mathrm{D}$ and $4.3 \%$ ended up in the potentially dangerous zone E (Fig. 2). In comparison, currently available semi-invasive glucose sensors show a mean correlation between sensor values and reference values of $\pm 90 \%$, a mean MAD of $\pm 11 \%$ and no values in the $\mathrm{D}$ and $\mathrm{E}$ zones [12-14].

\section{Retreat}

At the same time, Pendragon Medical was obviously dealing with internal and technical problems, according to two press-released shareholder letters from ProgressNow! invest, dated 2 and 3 September 2004 [15, 16]. Firstly, the need to solve the problem of unsuitability for a large group of patients due to strongly varying properties of the skin and underlying tissue was clearly pointed out in the pressreleased shareholder letter of 2 September 2004. Secondly, communication problems between the Pendragon board and its distributors in other countries were disclosed in the press-released shareholders letter of 3 September and were the subject of a broadcast on the Swiss television channel SFDRS on the same day. The Dutch launch symposium, planned for 2 September 2004, was unexpectedly cancelled on 30 August. Also on 2 September, Pendragon Medical and the Dutch importer discontinued their collaboration, and further entry of the sensor into the Dutch market was cancelled. Two renowned researchers in the field had stepped down from the Pendragon scientific advisory board on 25 July 2004 . When asked why, they claimed that confidentiality statements prohibited them from expanding on their reason for leaving. The patient undergoing treatment at our outpatient clinic demanded dissolution of the lease contract as well as a refund of his expenses, which he recently received.

On 28 January 2005 ProgressNow! reassessed the value of their investment $(16,309,540$ shares, cost price $€ 1.50$ each) to zero. The stock exchange rate decreased from $€ 76.80$ to $€ 23.40$ over the second half of 2004. On 4 February 2005, Pendragon Medical filed for bankruptcy.

\section{Lessons for the CE mark in Europe}

The premature marketing of the Pendra device seems to have been influenced by financial considerations and a very large demand for a good working non-invasive continuous glucose sensor. The results from our post-marketing validation study demonstrate poor accuracy of the current version of the Pendra device. The manufacturer has withdrawn the instrument from the market and acknowledged the limitations in patient suitability, the need of validation of prospective calibration and the considerable improvements needed to enable patient use.

Importantly, it appears that the current version of the Pendra device does not meet all CE essential requirements, for example alerting properly and measuring accurately. This casts doubts on its ability to supplement information obtained through standard glucose monitoring. However, the performance of the sensor during the $\mathrm{CE}$ valuation of conformity will remain undisclosed, as the results are the
Fig. 2 Clarke error grid with data pairs of Pendra glucose values and concomitant blood glucose values. Altogether, $78.4 \%$ of the paired values fell into the clinically acceptable zones $A$ and $B ; 6.5 \%$ were in zone $C ; 10.8 \%$ were in zone $D$; and $4.3 \%$ were in zone $E$

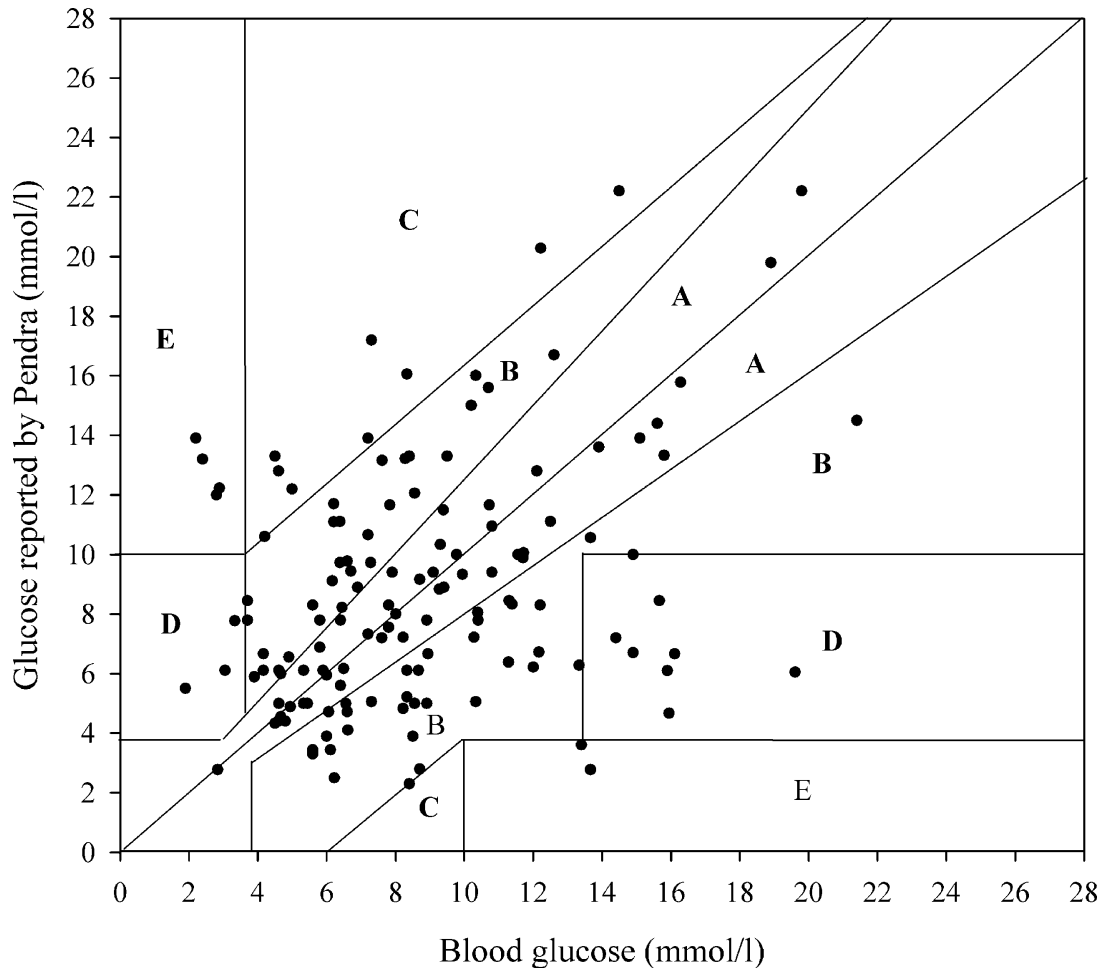


confidential property of the manufacturer and the authority. This lack of transparency prohibits a proper insight into the performance of a medical device sold directly to the consumer.

The question arises as to whether CE approval sufficiently guarantees ongoing reliability and safety of continuous glucose measuring devices such as the Pendra. Dysfunction of a sensor has clinically dangerous consequences, for example missing a hypoglycaemic episode or even inducing additional insulin administration when detecting hyperglycaemia while in fact hypoglycaemia is present. As such, a consensus for the requirements of glucose sensors, e.g. MAD $<25 \%$, needs to be developed and incorporated into the $\mathrm{CE}$ guidelines.

In 2001, a review by Koschinsky and Heinemann listed 25 companies that were developing glucose sensors at the time. Web research reveals the current existence of 37 companies and shows the disappearance of at least 16 companies listed in 2001 [17]. These data not only indicate a great turnover; they also highlight the potential reoccurrence of problems similar to those mentioned above if transparent requirements for glucose sensors are not developed.

In conclusion, to prevent disappointing investments from patients, but most of all to prevent patient exposure to potentially dangerous glucose sensors, more comprehensive and transparent requirements for CE approval need to be developed.

Acknowledgements We would like to acknowledge G. E. G. M. Storms and $\mathrm{H}$. van Leeuwen for their valuable contribution.

\section{References}

1. Bao J, Davis C (1992) Frequency domain impedance measurements of erythrocytes: constant phase angle impedance characteristics and a phase transition. Biophys J 61:1427-1434

2. Beving H, Eriksson G (1994) Dielectric spectroscopy of human blood. Eur J Surg [Suppl]:87-89

3. Zhao T, Lockner D (1993) Electrical impedance and erythrocyte sedimentation rate (ESR) of blood. Biochim Biophys Acta 1153:243-248
4. Pfutzner A, Caduff A, Larbig M, Schrepfer T, Forst T (2004) Impact of posture and fixation technique on impedance spectroscopy used for continuous and noninvasive glucose monitoring. Diabetes Technol Ther 6:435-441

5. Caduff A, Hirt E, Feldman Y, Ali Z, Heinemann L (2003) First human experiments with a novel non-invasive, non-optical continuous glucose monitoring system. Biosens Bioelectron 19:209-217

6. Scheffler M, Hirt E, Caduff A (2003) Wrist-wearable medical devices: technologies and applications. Med Device Technol $14: 26-30$

7. Authority for Official Publications of the European Community (1993) CE Guideline 93/42/EEG, regarding adjunctive medical devices. Appendix 1, Essential Requirements, The Netherlands, pp 22-28

8. Authority for Official Publications of the European Community (1993) CE Guideline, 93/42 EEG, regarding adjunctive medical devices. Appendix 2, EG-Declaration of Agreement, The Netherlands, pp 29-32

9. ProgressNow! invest AG to be listed on the SWS Swiss Exchange. http://www.progressnow.com/news/default.asp?page= $66 \&$ order $=$ news $\% 5$ Fstart $\% 5$ Fdate $\&$ cat, accessed 23 November 2004

10. Clarke WL, Cox D, Gonder-Frederick LA, Carter W, Pohl SL (1987) Evaluating clinical accuracy of systems for self-monitoring of blood glucose. Diabetes Care 10:622-628

11. Stock1 D, Dewitte K, Fierens C, Thienpont LM (2000) Evaluating clinical accuracy of systems for self-monitoring of blood glucose by error grid analysis: comment on constructing the "upper A-line." Diabetes Care 23:1711-1712

12. Guerci B, Floriot M, Bohme P et al (2003) Clinical performance of CGMS in type 1 diabetic patients treated by continuous subcutaneous insulin infusion using insulin analogs. Diabetes Care 26:582-589

13. Varalli M, Marelli G, Maran A et al (2003) A microdialysis technique for continuous subcutaneous glucose monitoring in diabetic patients (part 2). Biosens Bioelectron 18:899-905

14. Maran A, Crepaldi C, Tiengo A et al (2002) Continuous subcutaneous glucose monitoring in diabetic patients: a multicenter analysis. Diabetes Care 25:347-352

15. Shareholder letter of Pendragon Medical AG (2004) http:// www.progressnow.com/news/default.asp?page $=4 \&$ order $=$ news $\% 5$ Fstart $\% 5$ Fdate \&cat $=$, accessed 18 November 2004

16. Statement concerning the report in " 10 vor 10 " on 2 September 2004 on Pendragon Medical AG, a portfolio company of ProgressNow! invest AG (2004) http://www.progressnow.com/ news $/$ default.asp?page $=3$ \&order $=$ news $\% 5$ Fstart $\% 5$ Fdate $\&$ cat $=$, accessed 18 November 2004

17. Koschinsky T, Heinemann L (2001) Sensors for glucose monitoring: technical and clinical aspects. Diabetes Metab Res Rev $17: 113-123$ 